\title{
Energy transfers and spectral eddy viscosity in large-eddy simulations of homogeneous isotropic turbulence: Comparison of dynamic Smagorinsky and multiscale models over a range of discretizations
}

\author{
Thomas J. R. Hughes ${ }^{a}$ \\ The University of Texas at Austin, 201 East 24th Street, ACES 6.412, 1 University Station C0200, Austin, \\ Texas 78735-0027 \\ Garth N. Wells \\ Faculty of Civil Engineering and Geosciences, Delft University of Technology, Stevinweg 1, 2628 CN Delft, \\ The Netherlands \\ Alan A. Wray \\ NASA Ames Research Center, Moffett Field, California 94035
}

(Received 29 July 2003; accepted 13 July 2004; published online 5 October 2004)

\begin{abstract}
Energy transfers within large-eddy simulation (LES) and direct numerical simulation (DNS) grids are studied. The spectral eddy viscosity for conventional dynamic Smagorinsky and variational multiscale LES methods are compared with DNS results. Both models underestimate the DNS results for a very coarse LES, but the dynamic Smagorinsky model is significantly better. For moderately to well-refined LES, the dynamic Smagorinsky model overestimates the spectral eddy viscosity at low wave numbers. The multiscale model is in good agreement with DNS for these cases. The convergence of the multiscale model to the DNS with grid refinement is more rapid than for the dynamic Smagorinsky model. (C) 2004 American Institute of Physics.
\end{abstract}

[DOI: $10.1063 / 1.1789157]$

\section{INTRODUCTION}

The transfer of energy between different scales of motion in a turbulent flow has been studied widely for homogeneous isotropic turbulence. The flow of energy between different wave number modes is often expressed using the concept of an eddy viscosity. Heisenberg ${ }^{1}$ examined the loss of energy, in the Fourier domain, from modes within the interval $k=\left[0, k_{c}\right]$ to modes outside the interval. The transfer of energy from a mode below $k_{c}$ to modes beyond $k_{c}$ was represented by an additional viscosity, acting on the modes below $k_{c}$.

Theoretical studies of Kraichnan ${ }^{2}$ involved choosing an arbitrary wave number cutoff (analogous to a filter) and examining the form of the energy transfer to modes beyond the cutoff. Assuming infinite Reynolds number, Kraichnan ${ }^{2}$ identified the existence of a strong cusp in the spectral eddy viscosity near the cutoff wave number, and a plateau at low wave numbers (low relative to the cutoff). It was predicted that significant energy would be transferred from low wave numbers to wave numbers beyond the cutoff, owing to the presence of the plateau. However, the dominant mechanism of energy transfer was the cusp, which represented local interactions between modes below and near the cusp with modes having wave numbers no larger than twice the cutoff.

Following the theoretical studies of Kraichnan, ${ }^{2}$ the spectral eddy viscosity for homogeneous isotropic turbulence has been studied by direct numerical simulations (DNS). The spectral energy transfer can be calculated from a DNS data-

${ }^{a)}$ Author to whom correspondence should be addressed. base by introducing an arbitrary cutoff below the DNS limit of resolution., ${ }^{3,4}$ The eddy viscosity is calculated by examining the energy transfer from a mode below the cutoff to resolved modes beyond the cutoff. These calculations give an indication of the eddy viscosity that would be required for an effective large eddy simulation (LES) in which the cutoff corresponds to the LES limit of resolution. DNS results confirmed the presence of a cusp close to the cutoff wave number. However, no plateau at low wave numbers was found for moderate to high cutoffs in the low Reynolds number studies of Domaradzki, Liu, and Brachet, ${ }^{3}$ which indicate that no eddy viscosity on low wave number components in a LES would be required to replicate the DNS energy transfer.

McComb and Young ${ }^{4}$ studied energy transfers at somewhat higher Reynolds numbers. The spectral eddy viscosity was calculated from the a DNS of homogeneous isotropic turbulence with $256^{3}$ points, with a cutoff placed at a wave number below the limit of DNS resolution. The cutoff acts as an "explicit" filter, where all Fourier modes above a specified wave number are eliminated. Negligible energy transfer was observed from low wave number modes (relative to the explicit filter-the cutoff) to modes beyond the cutoff for higher values of the cutoff. Decreasing the cutoff wave number, the strength of the cusp increased. Only for the lowest cutoff was a plateau present.

Lesieur and colleagues have parametrized the plateau and cusp assuming a $k^{-5 / 3}$ range has developed at the cutoff (see Lesieur and Rogallo, ${ }^{5}$ Metais and Lesieur ${ }^{6}$ ), and shown a nonzero plateau which is close to that predicted by the test-field model ${ }^{2}$ and the eddy damped quasinormal Markovian (EDQNM) kinetic energy transfer model. ${ }^{7}$ We also wish 
to draw the reader's attention to the recent study of Cerutti, Meneveau, and $\mathrm{Knio}^{8}$ who have considered spectral eddy viscosities determined from experimental data.

Large-eddy simulations have been tested widely for homogeneous isotropic turbulence. The relative simplicity of the problem and the availability of well-resolved DNS results make it an attractive test bed. The variational multiscale method for the LES simulation of homogeneous isotropic turbulence was presented in Hughes et al. ${ }^{9}$ The velocity field was partitioned into coarse-scale (low wave number) and fine-scale (high wave number) components. The subgrid scale stress was then made a function of the fine-scale velocity field, and applied only to the fine-scale motions. Effectively, the LES model extracted energy only from high wave number modes. The method has a variational basis, as it relies on "projecting" the subgrid model onto the fine-scale motions, ${ }^{10}$ and it was shown to outperform conventional LES models in Hughes et al. ${ }^{9}$ It appeared that extracting energy only from high wave number modes led to better results than formulations which extract energy from all modes. Subsequent studies have also confirmed the good behavior of the variational multiscale method on a variety of problems: see Hughes, Oberai, and Mazzei, ${ }^{11}$ Winckelmans and Jeanmart, ${ }^{12}$ Oberai and Hughes, ${ }^{13}$ Farhat and Koobus, ${ }^{14}$ Jeanmart and Winckelmans, ${ }^{15}$ Holmen et al.,${ }^{16}$ Koobus and Farhat, ${ }^{17}$ Ramakrishnan and Collis. ${ }^{18-21}$

In this work, the spectral eddy viscosities for the conventional dynamic Smagorinsky model and the variational multiscale model are calculated and examined for a range of discretizations. The spectral eddy viscosity is decomposed into terms associated with Reynolds-type interactions, crossstress interactions, and the model. The results from the LES are compared with DNS data. Based on the results, conclusions are drawn as to the merits and deficiencies of the two LES models.

\section{FORMULATION}

The results presented in this work are simulations of homogeneous isotropic turbulence in a periodic box computed from DNS, the conventional dynamic Smagorinsky model, and the variational multiscale method. In this section, the formulation of the variational multiscale method for the simulation of homogeneous isotropic turbulence is briefly described.

In the multiscale formulation, the velocity field is partitioned into coarse-scale $(\overline{\mathbf{u}})$ and fine-scale $\left(\mathbf{u}^{\prime}\right)$ components. In a spectral context, the coarse-scale component is given by

$$
\overline{\mathbf{u}}=\sum_{|k|<\bar{k}} \hat{\mathbf{u}}_{k} \exp (i \mathbf{k} \cdot \mathbf{x}),
$$

where $k=|\mathbf{k}|$ and $\bar{k}$ defines the partition between coarse- and fine-scale components. The fine-scale motions then involve all resolved Fourier modes greater than and equal to $\bar{k}$.

$$
\mathbf{u}^{\prime}=\sum_{|k| \geqslant \bar{k}} \hat{\mathbf{u}}_{\mathbf{k}} \exp (i \mathbf{k} \cdot \mathbf{x})
$$

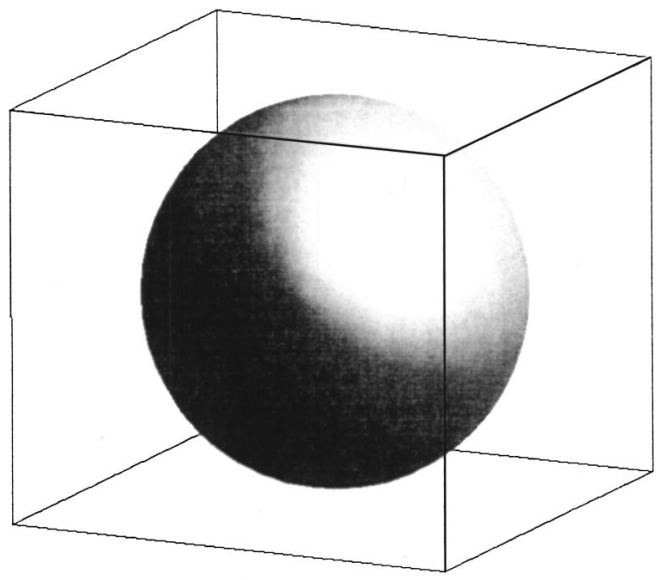

FIG. 1. Schematic illustration of the Fourier modes present in the simulations.

The Navier-Stokes momentum equation in the Fourier space with the multiscale subgrid model is given by ${ }^{9}$

$$
\left(\frac{d}{d t}+\nu|\mathbf{k}|^{2}\right) \hat{\mathbf{u}}_{\mathbf{k}}=-i \mathbf{k} \hat{p}_{\mathbf{k}}-\left[(\widehat{\mathbf{u} \otimes \mathbf{u}})_{\mathbf{k}}-H(k-\bar{k}) \hat{\boldsymbol{\tau}}_{k}\right],
$$

where $H$ is the Heaviside function $[H(x)=1$ if $x \geqslant 0$, otherwise $H(x)=0$ ]. Equation (3) implies that the subgrid stress term acts only on the fine-scale modes. Replacing $H(x)$ by 1 , a conventional LES model is recovered.

For conventional dynamic Smagorinsky and variational multiscale simulations, the Smagorinsky parameter $C_{s} \Delta$ is calculated using the dynamic procedure. ${ }^{22,23}$ The eddy viscosity at each point is then given by

$$
\nu_{T}=\left(C_{s} \Delta\right)^{2}\left|\nabla^{\mathrm{s}} \mathbf{u}\right|,
$$

where $\left|\nabla^{\mathrm{s}} \mathbf{u}\right|=\left(2 \nabla^{\mathrm{s}} \mathbf{u}: \nabla^{\mathrm{s}} \mathbf{u}\right)^{1 / 2}$. For the conventional dynamic Smagorinsky model, the subgrid stress is then calculated in the usual fashion and applied to all scales. For the multiscale model, the subgrid stress is calculated as

$$
\boldsymbol{\tau}=2 \nu_{T} \nabla^{\mathrm{s}} \mathbf{u}^{\prime},
$$

which involves the gradient of the fine-scale velocity field. Hughes et al. ${ }^{9}$ used the constant Smagorinsky model in combination with the multiscale method. Note however that here, the term $C_{s} \Delta$ is calculated using the dynamic procedure.

\section{NUMERICAL RESULTS}

The numerical simulations are performed in a cube with a side length of $2 \pi$ and periodic boundary conditions. A schematic illustration of the cube is presented in Fig. 1. The cube denotes a lattice in wave-vector space and each point in the lattice corresponds to a resolved Fourier mode. If the edge of the cube has length $2 k^{\prime}$, then the wave number of the highest Fourier mode present is $\sqrt{3} k^{\prime}$. The sphere depicts the internal cutoff $\bar{k}$ which is taken to be $\sqrt{3} k^{\prime} / 2$. In the multiscale cases, the interior of the sphere corresponds to the coarse-scale subspace, whereas the complement corresponds to the fine-scale subspace. The coarse-scale subspace is thus $\approx 34 \%$ of the total space. 


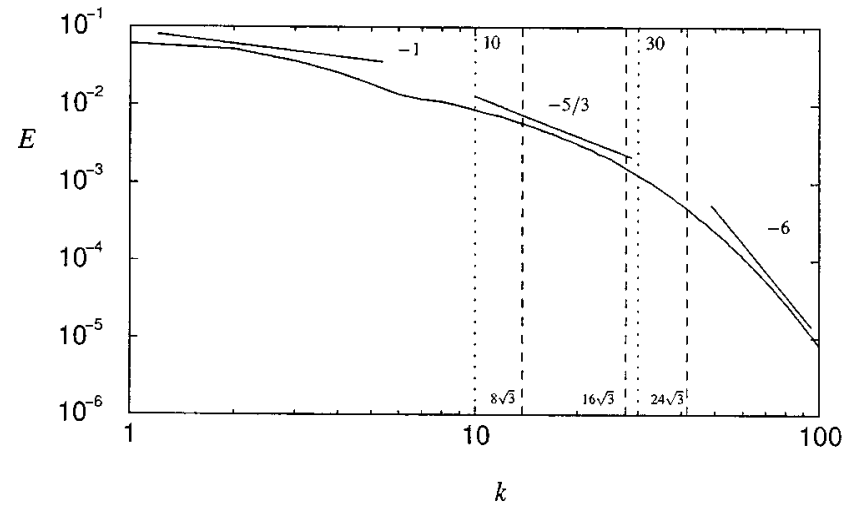

(a)

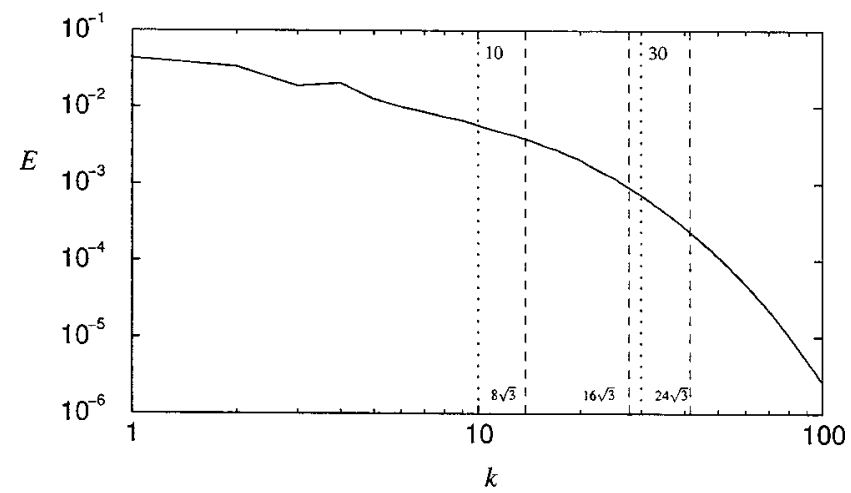

(b)

FIG. 2. DNS energy spectrum for (a) initial $256^{3}$ DNS; and (b) final $160^{3}$ DNS energy spectrum advanced from $256^{3}$ initial DNS for one large-eddy turnover time. The various values of $\bar{k}$ are indicated by the dashed lines, and the $-5 / 3$ region is bounded approximately by the dotted lines $(k=10$ and $k=30$ ).

The numerical results are calculated using a pseudospectral method. Nonlinear terms are integrated using a four-step, third-order Runge-Kutta scheme and terms due to molecular viscosity are integrated using the integrating factor method. Nonlinear convective terms are de-aliased using the $3 / 2$ rule. Terms relating to the LES model are not de-aliased. For all calculations, the initial flow field is taken from a welldeveloped DNS with $256^{3}$ Fourier modes. The initial energy spectrum, together with relevant resolution limits for following simulations, is shown in Fig. 2(a). Note that three regimes of the spectrum are identified in Fig. 2(a), corresponding to approximate slopes of $-1,-5 / 3$, and -6 . The $-5 / 3$ regime extends from about $k=10$ to $k=30$. Beyond $k=30$, the slope asymptotes smoothly to -6 .

All plots of spectral eddy viscosity have been presented on the same scale to allow quantitative comparisons.

\section{A. DNS results}

The flow is advanced for approximately one large-eddy turnover time at a resolution of $160^{3}$ Fourier modes. The energy spectrum is shown in Fig. 2(b). To place LES results in context, the spectral energy transfer for direct numerical simulations with various cutoffs is first examined.

The energy transfers, expressed as spectral eddy viscosities, due to Reynolds stress interactions, cross-stress interac-

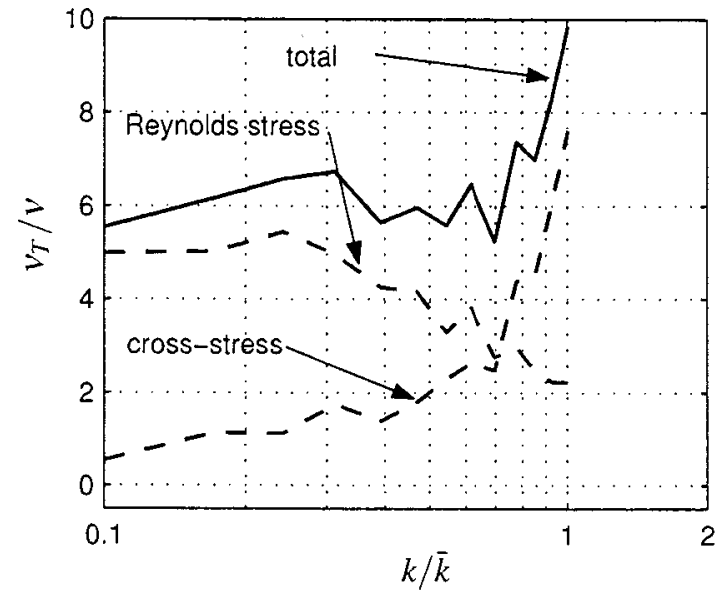

(a) $\bar{k}=8 \sqrt{3}$

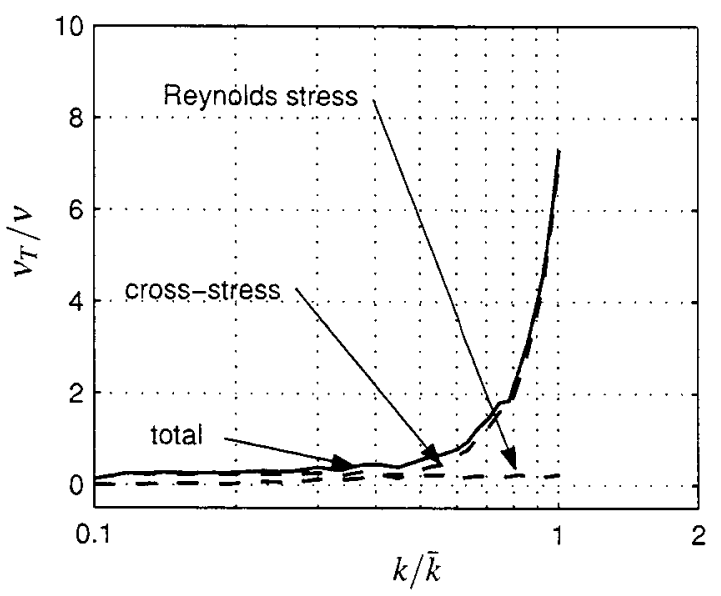

(b) $\bar{k}=16 \sqrt{3}$

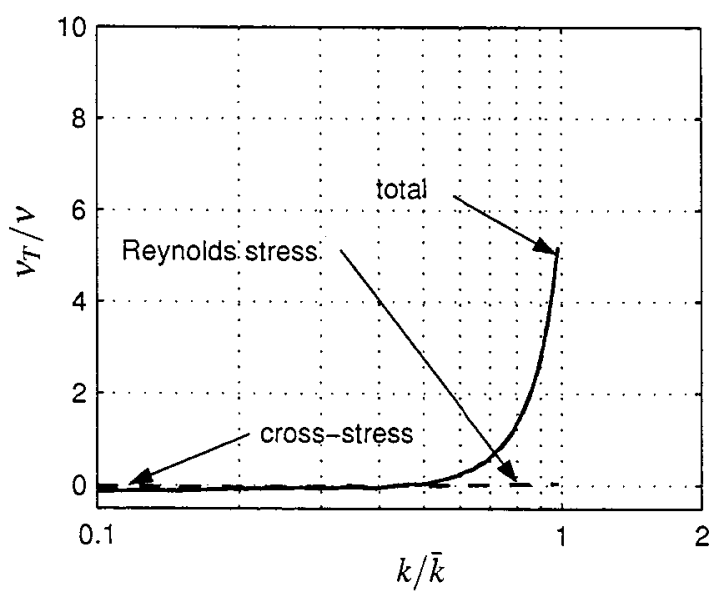

(c) $\bar{k}=24 \sqrt{3}$

FIG. 3. Spectral energy transfer for a range of cutoffs for $160^{3}$ DNS.

tions, and the sum of the two are shown in Fig. 3. The spectral eddy viscosities are shown in Fig. 3 with three different spherical cutoffs: $\bar{k}=8 \sqrt{3} ; 16 \sqrt{3}$; and $24 \sqrt{3}$. These values of $\bar{k}$ are identified in Fig. 2. The first cutoff, $\bar{k}=8 \sqrt{3}$, is at the beginning of the $k^{-5 / 3}$ regime; the second, $\bar{k}=16 \sqrt{3}$, is close to the end of it; and the third, $\bar{k}=24 \sqrt{3}$, is within the dissipation regime. The calculated eddy viscosity represents the transfer of energy from modes below $\bar{k}$ to modes between $\bar{k}$ and the DNS limit of resolution. 
From Fig. 3, a significant plateau in the spectral eddy viscosity clearly exists for the $\bar{k}=8 \sqrt{3}$ case at low wave numbers, and the cusp is weak. This case is representative of the energy extraction that would be required for a very coarse LES. Increasing $\bar{k}$ to $16 \sqrt{3}$, the spectral eddy viscosity takes on the classic plateau-cusp profile. There is a small plateau at low wave numbers which is due to Reynolds stress interactions. Cross-stress interactions lead to the typical cusp as $\bar{k}$ is approached. Examining the spectral eddy viscosity for $\bar{k}$ $=24 \sqrt{3}$, Reynolds stress interactions are insignificant across all wave numbers, and energy transfers are primarily due to cross-stress interactions. In the absence of a plateau, the energy transfer from low wave number modes to modes beyond $\bar{k}$ is negligible.

Remark. The case of $\bar{k}=8 \sqrt{3}$ is coarse enough to be representative of the inviscid case. This is confirmed by computing the value of the eddy viscosity plateau from the parametrization given by Lesieur and Rogallo ${ }^{5}$ and Metais and Lesieur, ${ }^{6}$ which is predicated on the cutoff being within the $k^{-5 / 3}$ range. In this case, $\nu_{T} / \nu=0.267 \sqrt{E(\bar{k}) / \bar{k}} / \nu \approx 0.005 / \nu$ $=5.3$. This value is comparable to the computed plateau, $\nu_{T} / \nu \approx 0.006 / \nu=6.3$, but slightly less which is consistent with the fact that the slope at $\bar{k}$ is in the range of $k^{-m}$ with $m$ slightly less than $5 / 3$. Inviscid cases have been thoroughly studied in Lesieur and Rogallo ${ }^{5}$ and Metais and Lesieur, ${ }^{6}$ confirming the test-field model of Kraichnan ${ }^{2}$ and the EDQNM calculations of Lesieur. ${ }^{7}$ For the cases $\bar{k}=16 \sqrt{3}$ and $\bar{k}=24 \sqrt{3}$, the Lesieur-Metais-Rogallo parametrization gives values of $\nu_{T} / \nu$ much higher than those computed, which is consistent with the fact that for these cases the cutoff is in the range of $k^{-m}$ with $m>5 / 3$. In this situation, the "spectral dynamic" model (see Lesieur ${ }^{24}$ ), which is applicable for $m>5 / 3$, may be utilized to obtain more accurate estimates of $\nu_{T} / \nu$.

\section{B. LES energy transfers: Addition of the model component}

The spectral eddy viscosity is now examined for the dynamic Smagorinsky and multiscale LES models. Simulations are performed with $32^{3}, 64^{3}$, and $96^{3}$ Fourier modes. For each LES, $\bar{k}$ is placed at half the limit of resolution, namely, $\bar{k}=8 \sqrt{3}, 16 \sqrt{3}$, and $24 \sqrt{3}$, respectively, which is the same placement as for the DNS spectral eddy viscosity calculations. We consider these LES to be very coarse, moderately refined, and well refined, respectively. These characterizations are supported by the energy spectra, shown in Fig. 2. The spectral eddy viscosity is decomposed into components corresponding to Reynolds stress interactions, cross-stress interactions, and the LES model. The sum of these components is compared to the DNS total spectral eddy viscosity for the same $\bar{k}$. Ideally, the addition of the model contribution to the sum of the Reynolds and cross-stress spectral eddy viscosities will bring the total spectral eddy viscosity to that for the DNS. In the following figures, the relevant DNS

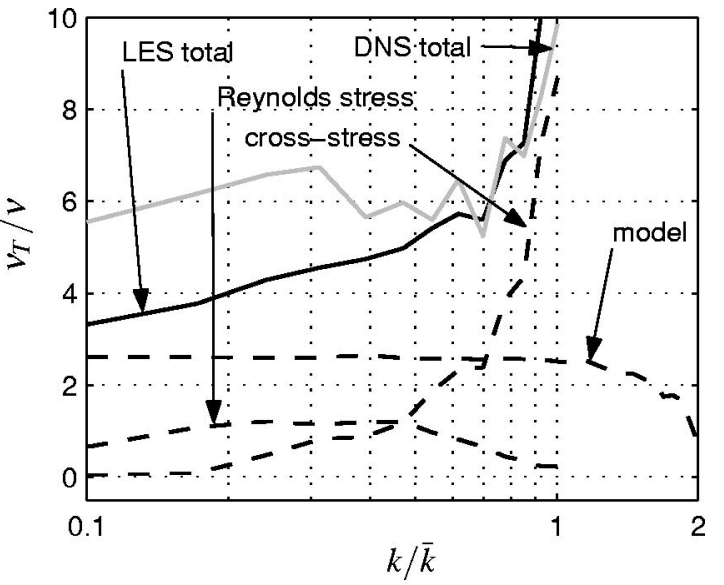

(a) $32^{3}, \bar{k}=8 \sqrt{3}$

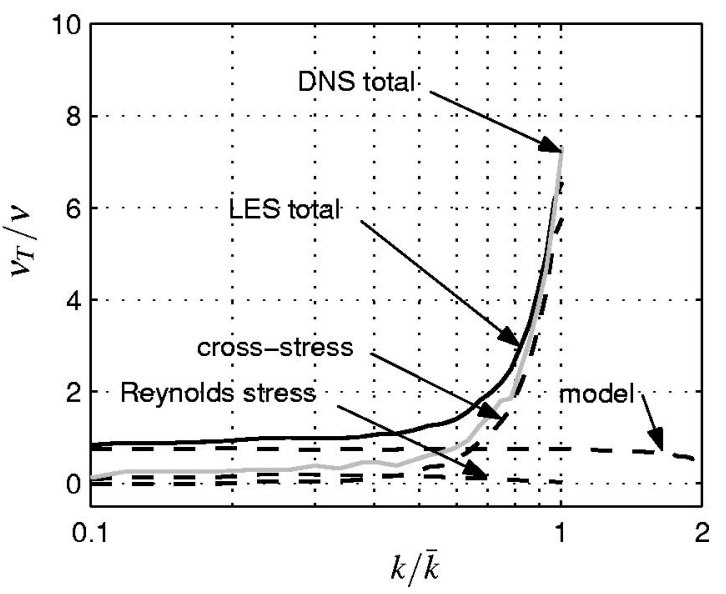

(b) $64^{3}, \bar{k}=16 \sqrt{3}$

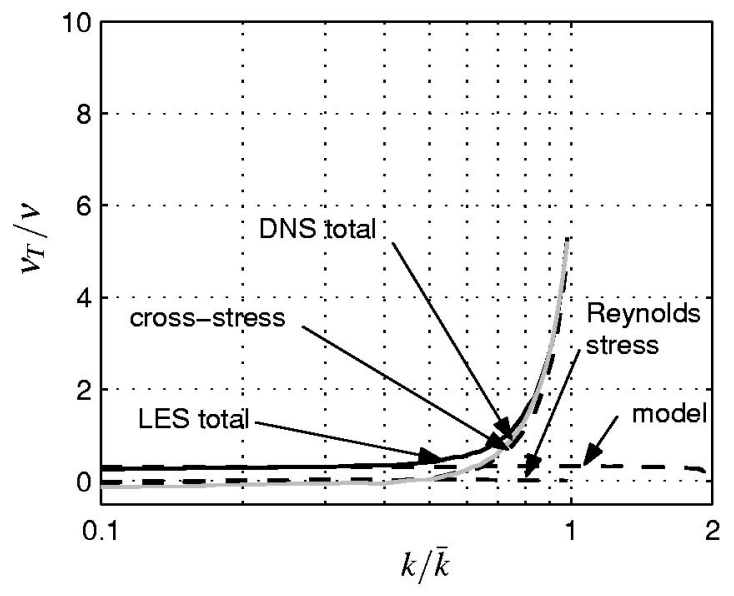

(c) $96^{3}, \bar{k}=24 \sqrt{3}$

FIG. 4. Dynamic Smagorinsky model: spectral energy transfer for a range of discretizations and cutoffs.

spectral eddy viscosity is shown in gray. For the LES results, the model eddy viscosity is shown up to the limit of LES resolution $(k / \bar{k}=2)$.

The spectral eddy viscosity for a series of LES with the conventional dynamic Smagorinsky model is shown in Fig. 4. For the least-well-resolved case [Fig. 4(a)], it is clear that 
LES energy transfers due to Reynolds and cross-stress interactions are too small compared to the DNS result because there are too few modes between $\bar{k}=8 \sqrt{3}$ and the limit of resolution. The model compensates somewhat for this deficiency in the low wave number region, but the model supplements the Reynolds and cross-stress interactions insufficiently to reach the DNS dissipation level. For the better resolved LES [Figs. 4(b) and 4(c)], the spectral eddy viscosity due to Reynolds-type interactions is small, as it was in the DNS case. The cross-stress contribution to the energy transfer is also small at low wave numbers, but exhibits the cusp near $\bar{k}$. Generally, the total spectral eddy viscosity for the two better-resolved dynamic LES cases match the DNS spectral eddy viscosity well close to $\bar{k}$. However, the differences are significant at lower wave numbers. The deviation in the spectral eddy viscosity from the DNS result is attributable to the contribution of the model at low wave numbers. While the DNS eddy viscosity is small to negligible at low wave numbers, the model contribution is significant. Curiously, for all cases, the model contribution is nearly constant across all wave numbers. It can be summarized from Fig. 4 that the dynamic Smagorinsky model introduces spurious dissipation at low wave numbers for moderately to well-resolved LES.

The spectral eddy viscosity for a series of multiscale LES is shown in Fig. 5. The discretizations and partitions are the same as those for the dynamic Smagorinsky model. Consistent with the multiscale concept, the model eddy viscosity is zero below $\bar{k}$, and acts only beyond $\bar{k}$, as can be seen in Fig. 5. For the coarsest discretization [Fig. 5(a)], the multiscale model is unable to represent the significant plateau at low wave numbers. For the better resolved cases [Figs. 5(b) and 5(c)], the multiscale results correspond very well with the DNS results. The absence of a model acting at low wave numbers avoids the spurious plateau, leading to results which are in good agreement with the DNS results.

The spectra for the multiscale LES are compared to the $160^{3}$ DNS at the end of the simulation time in Fig. 6. As expected, based on the previous results, the spectra for the two better resolved multiscale LES match the DNS spectrum up to the $\bar{k}$ cutoff very well. In the coarse case, the LES spectrum lies above the DNS, which is consistent with the fact that the spectral eddy viscosity is significantly underestimated in this case. In all multiscale cases, the LES spectra drops abruptly below that of the DNS for $k>\bar{k}$. Note that the LES spectra are shown up to the limit of resolution.

Remark. It is important to note that there are differences in the multiscale method utilized in this paper and that employed in our initiatory study. ${ }^{9}$ In Hughes et al.,${ }^{9} \bar{k}=k^{\prime} / 2$ and a static Smagorinsky-type model was used in the fine scales. Here, $\bar{k}=\sqrt{3} k^{\prime} / 2$ and a dynamic Smagorinsky-type model is used. In the present case $\bar{k}$ is quite close to $k^{\prime}$. The abrupt drop in the energy spectrum beyond $\bar{k}$ noted here (see Fig. 6) was not observed in our earlier study when $\bar{k}$ was smaller compared with $k^{\prime}$. No effort has been made yet to determine optimal values of $\bar{k}$ for homogeneous isotropic flows. An initiatory study of the sensitivity of results to the ratio $\bar{k} / k^{\prime}$,

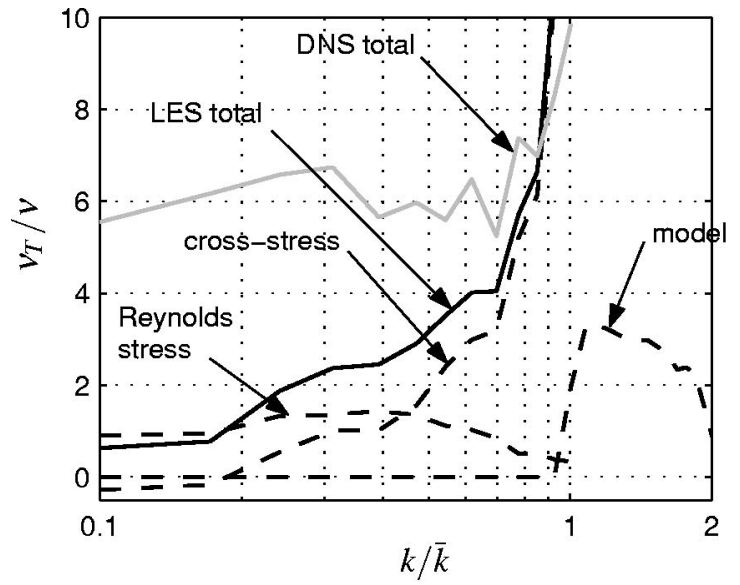

(a) $32^{3}, \bar{k}=8 \sqrt{3}$

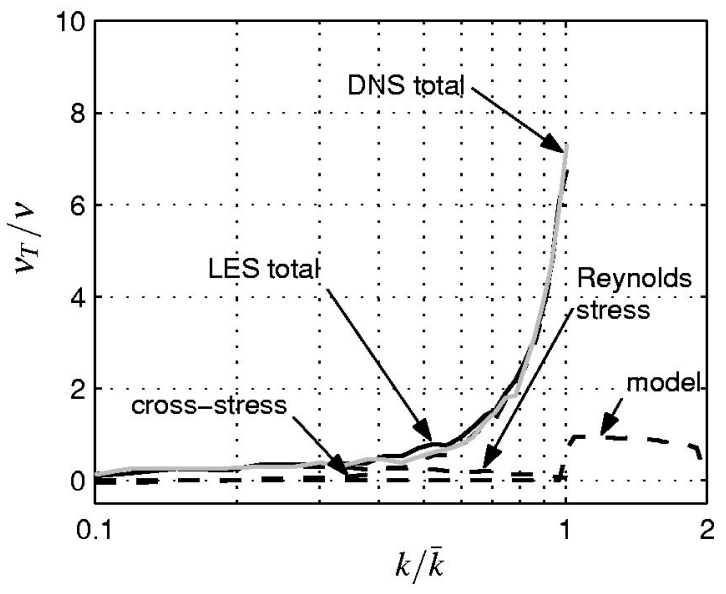

(b) $64^{3}, \bar{k}=16 \sqrt{3}$

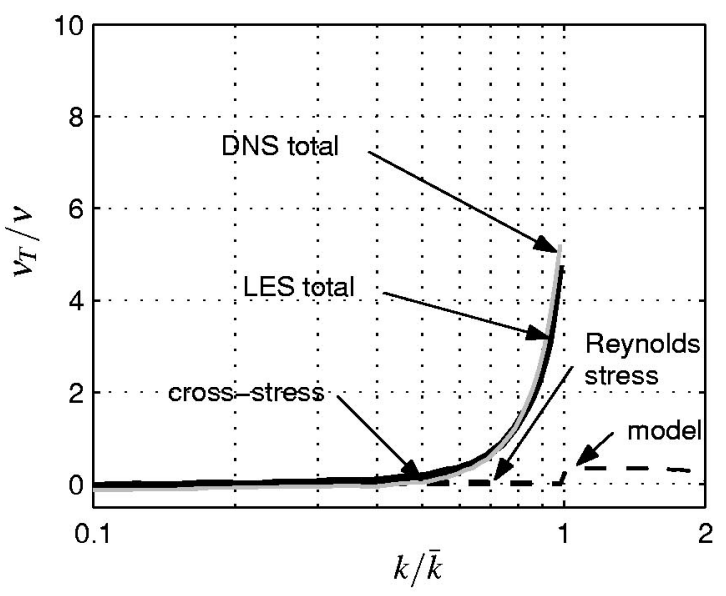

(c) $96^{3}, \bar{k}=24 \sqrt{3}$

FIG. 5. Multiscale model: spectral energy transfer for a range of discretizations and cutoffs.

with $k^{\prime}$ fixed, for channel flows is presented in Holmen et al. ${ }^{16}$ There it was observed that smaller ratios of $\bar{k} / k^{\prime}$ $(\approx 0.5)$ better suited the dynamic multiscale model and larger ratios of $\bar{k} / k^{\prime}(\approx 0.7)$ performed better for the static multiscale model. It has not yet been determined whether this trend is generally applicable. 

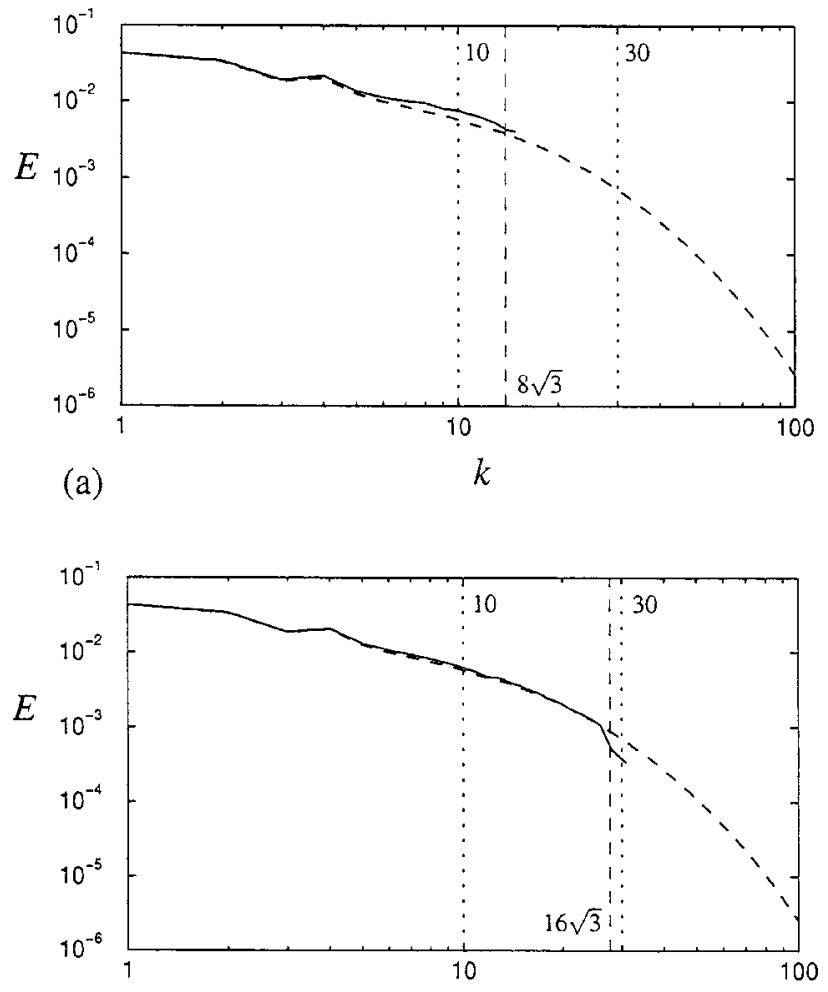

(b)

$k$

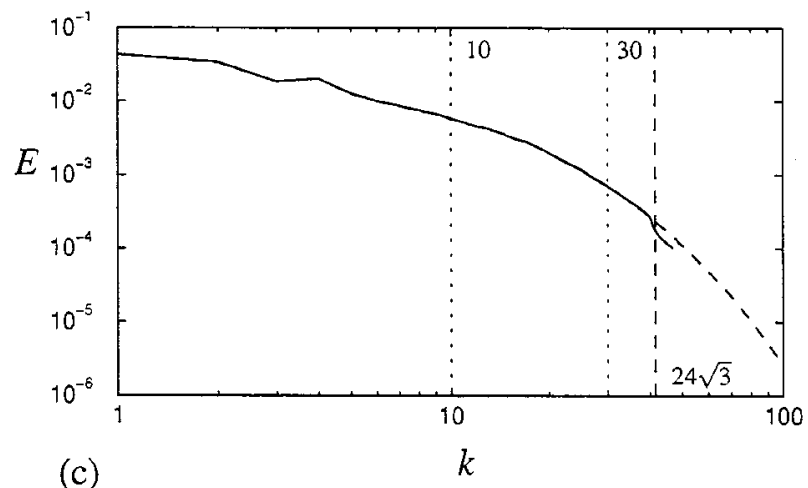

FIG. 6. Multiscale energy spectra (solid line) after one eddy turnover for (a) $\bar{k}=8 \sqrt{3}, k^{\prime}=15$; (b) $\bar{k}=16 \sqrt{3}, k^{\prime}=31$; and (c) $\bar{k}=24 \sqrt{3}, k^{\prime}=47$. The $160^{3}$ DNS spectrum, given by the dashed line, is provided for comparison. The $-5 / 3$ region is bounded approximately by the dotted lines ( $k=10$ and $k=30)$.

\section{Comparison of the dynamic and multiscale models}

From the preceding results, it is clear that, for the better resolved calculations, the spectral eddy viscosity for the multiscale case is closer to the DNS results than the dynamic Smagorinsky case. To examine the differences between the dynamic Smagorinsky and multiscale models, the total spectral eddy viscosity (Reynolds stress plus cross-stress plus model contribution) for the two LES models is compared in Fig. 7. As a reference, the DNS total is included. Figure 7 shows the total eddy viscosity for the two LES models and the DNS for $\bar{k}=8 \sqrt{3}, 16 \sqrt{3}$, and $24 \sqrt{3}$. For the coarsest discretization, both LES models underestimate the spectral eddy viscosity across all wave numbers, although the contribution of the dynamic model to the low-mode dissipation leads to a

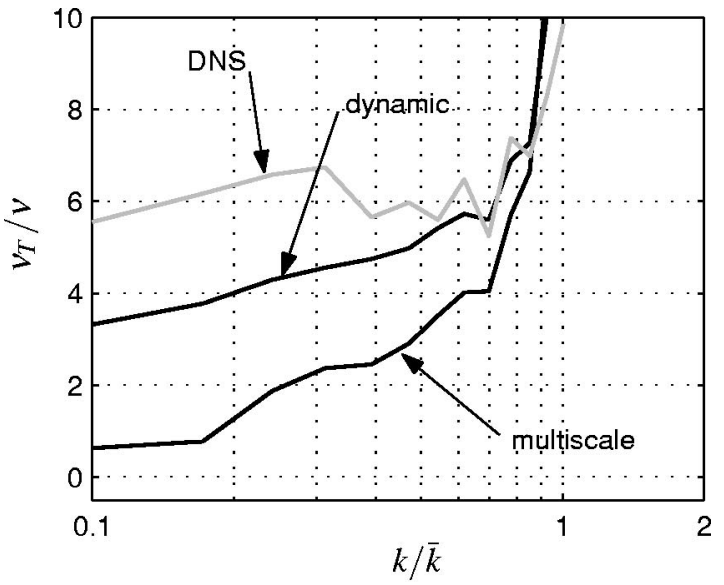

(a) $32^{3}, \bar{k}=8 \sqrt{3}$

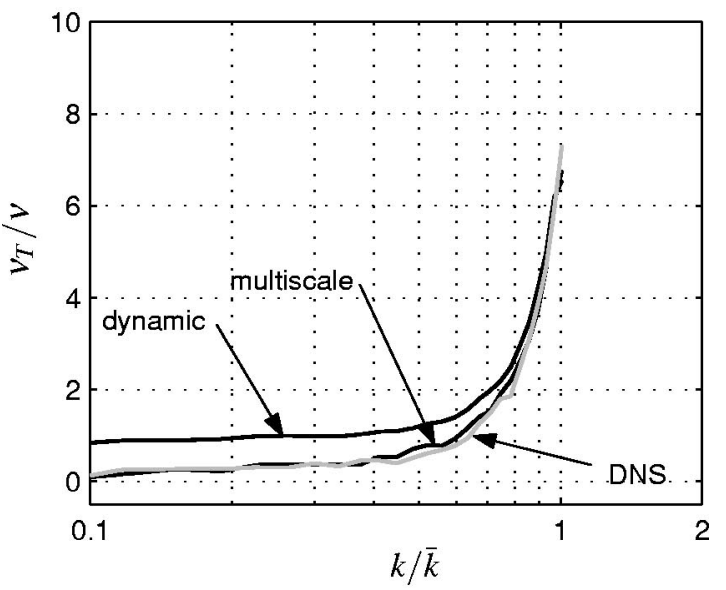

(b) $64^{3}, \bar{k}=16 \sqrt{3}$

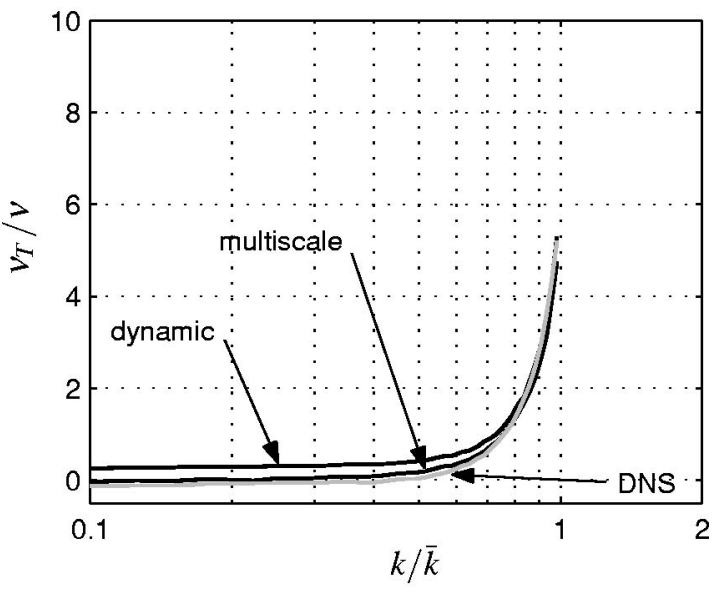

(c) $96^{3}, \bar{k}=24 \sqrt{3}$

FIG. 7. Comparison of the total spectral eddy viscosity for the LES models.

better result than with the multiscale model. For the better resolved cases, the multiscale model is in better agreement with the DNS, across all wave numbers.

For each LES case and the DNS, the spectral eddy viscosity is now decomposed into its Reynolds-type and crossstress components. Figure 8 shows the Reynolds stress contribution to the spectral eddy viscosity. Clearly, for the coarse discretization the Reynolds stress contribution for the LES is very small compared to the DNS. However, upon refining the 


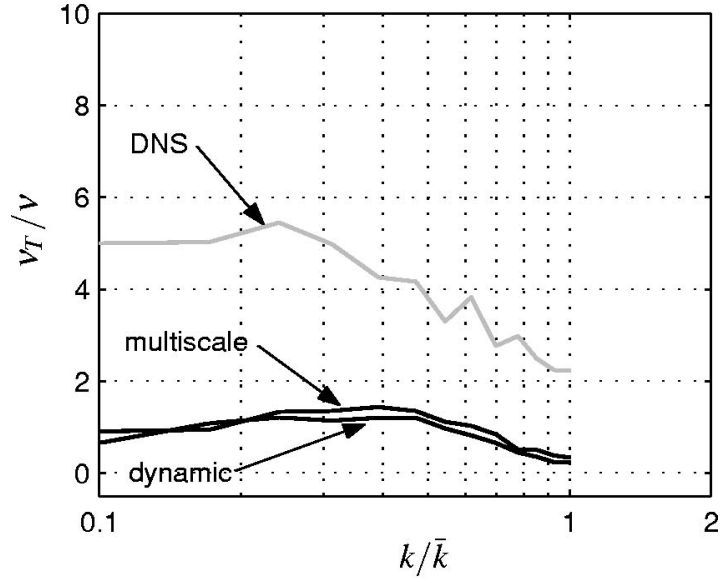

(a) $32^{3}, \bar{k}=8 \sqrt{3}$

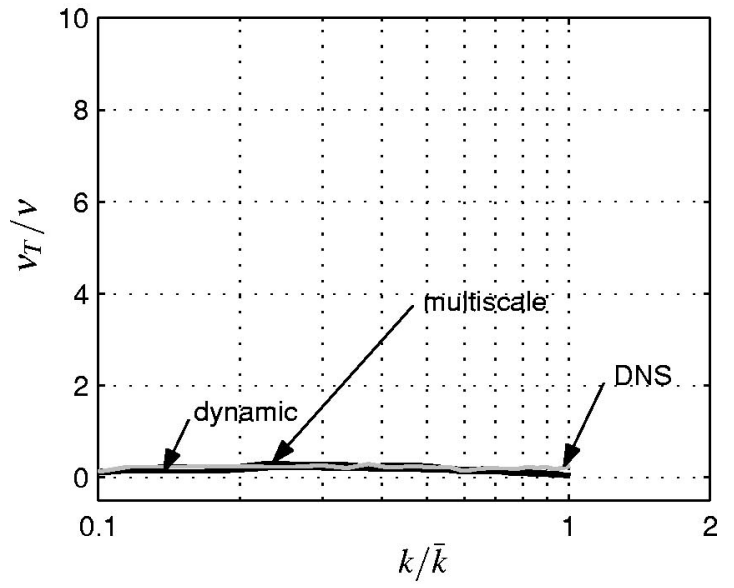

(b) $64^{3}, \bar{k}=16 \sqrt{3}$

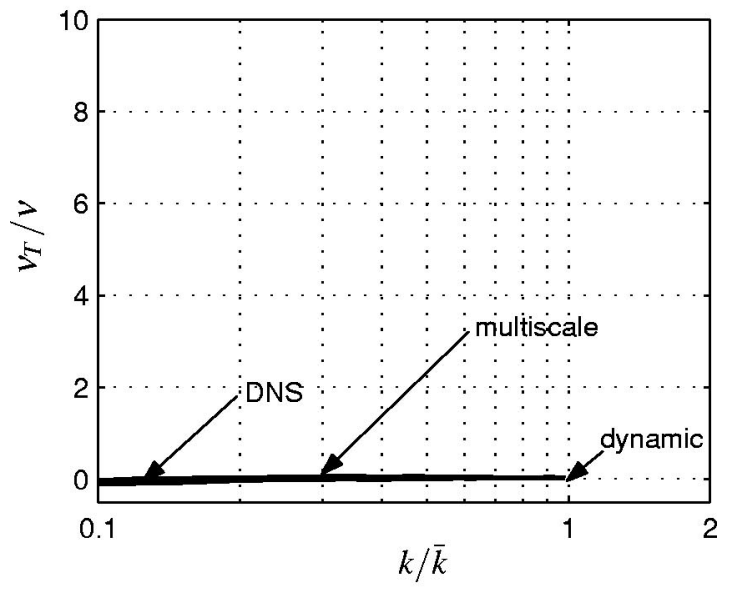

(c) $96^{3}, \bar{k}=24 \sqrt{3}$

FIG. 8. Reynolds stress contribution to the spectral eddy viscosity for LES.

discretization, the Reynolds-stress component becomes extremely small for all cases and even slightly negative for the finest DNS. Figure 8 shows how rapidly the low wave number plateau (which is due to Reynolds stresses) vanishes with improved resolution.

Figure 9 shows the spectral energy transfer attributable to cross-stress interactions. For all cases, both LES formulations are in reasonable agreement with the DNS result. For the coarsest resolution, the low wave number spectral eddy

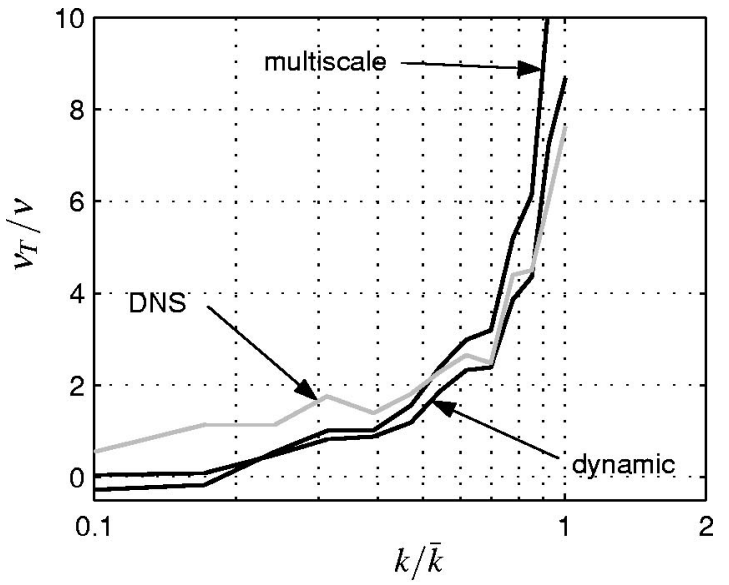

(a) $32^{3}, \bar{k}=8 \sqrt{3}$

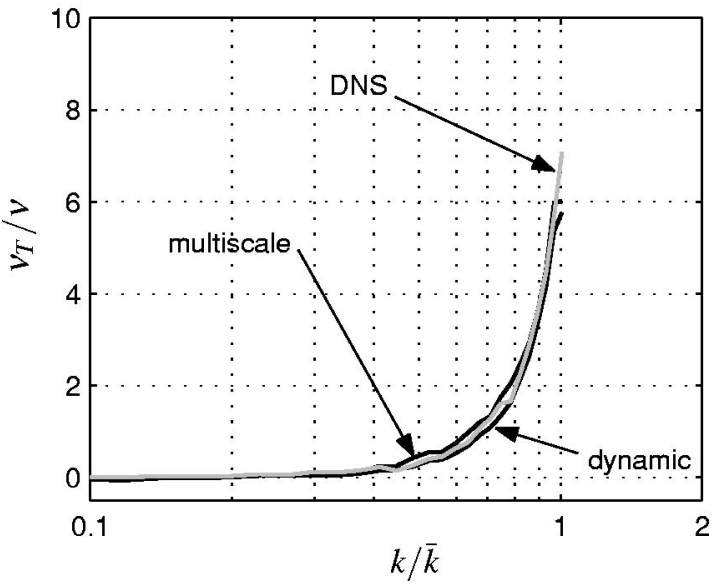

(b) $64^{3}, \bar{k}=16 \sqrt{3}$

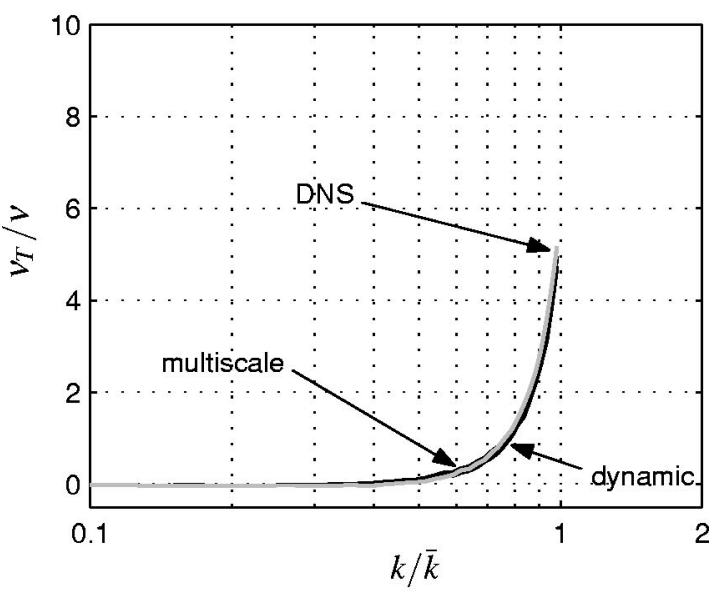

(c) $96^{3}, \bar{k}=24 \sqrt{3}$

FIG. 9. Cross-stress contribution to the spectral eddy viscosity for LES.

viscosity due to cross-stress interactions is low relative to the eddy viscosity due to Reynolds stress interactions [Fig. 8(a)]. For the better resolved cases, the low-wave number eddy viscosity due to cross-stress interactions is effectively zero for all cases.

The spectral eddy viscosity due to Reynolds-type and cross-stress interactions for the conventional dynamic Smagorinsky model is reasonably close to the corresponding quantities from the DNS. However, the total spectral eddy 


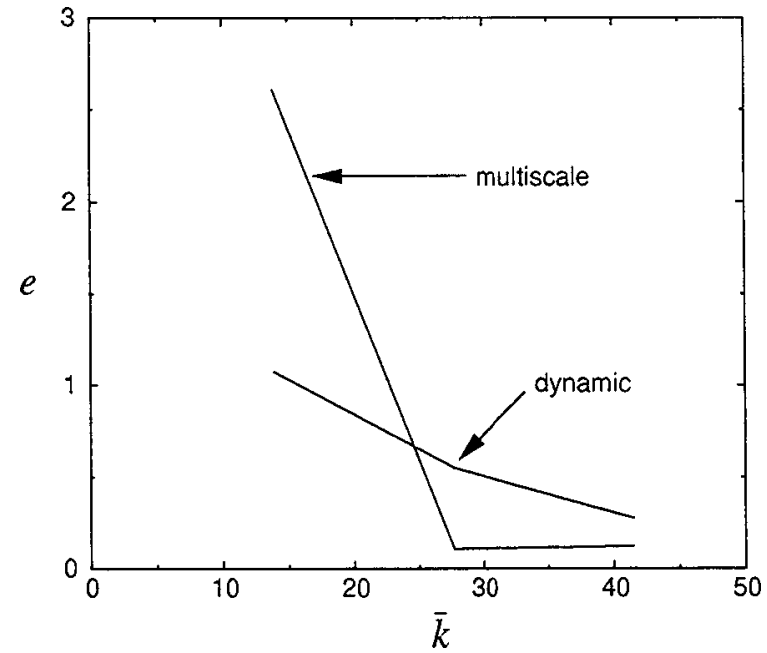

FIG. 10. Spectral eddy viscosity error as a function of $\bar{k}$.

viscosity deviates from the DNS results. For moderately to well-resolved cases, the error is introduced by the model, which is ironically introduced to improve the representation. The model introduces a low wave number plateau to the spectral eddy viscosity which is not present in the DNS results.

\section{Convergence}

A global measure of the error in the eddy viscosity, corresponding to Fig. 7, is given by

$$
e=\int_{0}^{1}\left|\nu_{T}^{\mathrm{LES}}(\widetilde{k})-\nu_{T}^{\mathrm{DNS}}(\tilde{k})\right| d \widetilde{k} / \nu,
$$

where

$$
\tilde{k}=\frac{k}{k}
$$

This quantity is presented in Fig. 10 for all the cases studied. For the coarsest discretization, the error is smaller for the conventional dynamic Smagorinsky model, whereas the multiscale model is more accurate for the better resolved simulations.

\section{CONCLUSIONS}

The spectral eddy viscosities for a conventional dynamic Smagorinsky LES model and the multiscale LES model have been examined and compared to DNS results.

For the coarsest discretization, the dynamic Smagorinsky model spectral eddy viscosity is closer to that for the DNS than the multiscale model, owing to the introduction of eddy viscosity to the low modes. For moderately to wellrefined simulations, the conventional dynamic Smagorinsky model overestimates the eddy viscosity in low wave number modes. The eddy viscosity introduced by the dynamic Smagorinsky model is nearly constant across all wave numbers. While the model has the ability to adapt itself to a flow, its distribution is nearly uniform across the Fourier modes and it appears unable to partition itself appropriately to the different scales of motion in the flow.

For the moderately- to well-refined cases, the multiscale spectral eddy viscosity more accurately approximates that for the DNS than the conventional dynamic Smagorinsky model. In particular, as the discretization is refined, convergence to the DNS is more rapid than for the conventional dynamic Smagorinsky model. However, the multiscale model significantly underestimates energy transfers from the low-wave number part of the spectrum to unresolved modes in the coarsest case. This is important because practical calculations with LES may behave in a similar fashion to this case.

These results provide additional understanding of the behavior of the multiscale model and the conventional dynamic Smagorinsky model for homogeneous isotropic flows and suggest research directions that may lead to further improvements. In the case of the multiscale method, the representation of the short-range action of the cusp near the cutoff by a fine-scale eddy viscosity model seems to be a simple and adequately accurate procedure. The issue of how to model the plateau, that is, the long-range transfers from the low wave number part of the spectrum to the unresolved modes, appears to be a more delicate matter. An eddy viscosity mechanism in the low wave number regime is unlikely to be the answer because it is well-known from standard numerical analysis theory that artificial viscosities acting on low wave number components preclude higher-order, and, in particular, spectral accuracy. Furthermore, one needs to realize that the physical mechanism of long-range transfers is not a viscous phenomenon. How to go about this appears to be an open question at this juncture.

\section{ACKNOWLEDGMENTS}

T.J.R.H. was supported by ONR Grant No. 00014-03-10263, NASA Ames Research Center Grant No. NAG2-1604, and Sandia National Laboratories Grant No. A0340.0. G.N.W. was supported by the Netherlands Organisation for Scientific Research (NWO) and the J. Tinsley Oden Faculty Research Program, Institute for Computational Engineering and Sciences, The University of Texas at Austin. The authors gratefully acknowledge the support provided by these organizations.

\footnotetext{
${ }^{1}$ W. Heisenberg, "On the theory of statistical and isotropic turbulence," Proc. R. Soc. London, Ser. A 195, 402 (1948).

${ }^{2}$ R. H. Kraichnan, "Eddy viscosity in two and three dimensions," J. Atmos. Sci. 33, 1521 (1976).

${ }^{3}$ J. A. Domaradzki, W. Liu, and M. E. Brachet, "An analysis of subgridscale interactions in numerically simulated isotropic turbulence," Phys. Fluids A 5, 1747 (1993).

${ }^{4} \mathrm{D}$. McComb and A. Young, "Explicit-scales projections of the partitioned non-linear term in direct numerical simulation of the Navier-Stokes equation," in Second Monte Verita Colloquium on Fundamental Problematic Issues in Fluid Turbulence, Ascona, Switzerland, 1998.

${ }^{5}$ M. Lesieur and R. Rogallo, "Large-eddy simulation of passive scalar diffusion in isotropic turbulence," Phys. Fluids A 1, 718 (1989).

${ }^{6} \mathrm{O}$. Metais and M. Lesieur, "Spectral large-eddy simulation of isotropic and stably stratified turbulence," J. Fluid Mech. 239, 157 (1992).

${ }^{7}$ M. Lesieur, Turbulence in Fluids, 3rd ed. (Kluwer, Dordrecht, 1997).
} 
${ }^{8}$ S. Cerutti, C. Meneveau, and O. M. Knio, "Spectral and hyper eddy viscosity in high-Reynolds-number turbulence," J. Fluid Mech. 421, 307 (2000).

${ }^{9}$ T. J. R. Hughes, L. Mazzei, A. A. Oberai, and A. A. Wray, "The multiscale formulation of large eddy simulation: Decay of homogeneous isotropic turbulence," Phys. Fluids 13, 505 (2001).

${ }^{10}$ T. J. R. Hughes, L. Mazzei, and K. E. Jansen, "Large eddy simulation and the variational multiscale method," Comput. Vis. Sci. 3, 47 (2000).

${ }^{11}$ T. J. R. Hughes, A. A. Oberai, and L. Mazzei, "Large eddy simulation of turbulent channel flows by the variational multiscale method," Phys. Fluids 13, 1784 (2001).

${ }^{12} \mathrm{G}$. S. Winckelmans and H. Jeanmart, "Assessment of some models for LES without/with explicit-filtering," in Direct and Large-Eddy Simulation $I V$, ERCOFTAC Series, edited by B. J. Geurts, R. Friedrich, and O. Métais (Kluwer, Dordrecht, 2001), pp. 55-66.

${ }^{13}$ A. A. Oberai and T. J. R. Hughes, "The variational multiscale formulation of LES: Channel flow at $\operatorname{Re}_{\tau}=590$," AIAA Paper No. 2002-1056, Reno, Nevada, 14-17 January 2002.

${ }^{14} \mathrm{C}$. Farhat and B. Koobus, "Finite volume discretization on unstructured meshes of the multiscale formulation of large eddy simulations," in Proceedings of the Fifth World Congress on Computational Mechanics ( WCCM V), edited by H. A. Mang, F. G. Rammerstorfer, and J. Eberhardsteiner (Vienna University of Technology, Vienna, Austria, 2002).

${ }^{15} \mathrm{H}$. Jeanmart and G. S. Winckelmans, "Comparison of recent dynamic subgrid-scale models in turbulent channel flow," in Proceedings of the Summer Program 2002, edited by P. Bradshaw (Center for Turbulence
Research, Stanford University, Stanford, California, 2002).

${ }^{16}$ J. Holmen, T. J. R. Hughes, A. A. Oberai, and G. N. Wells, "Sensitivity of the scale partition for variational multiscale LES of channel flow," Phys. Fluids 16, 824 (2004).

${ }^{17}$ B. Koobus and C. Farhat, "A variational multiscale method for the large eddy simulation of compressible turbulent flows on unstructured meshesApplication to vortex shedding," Comput. Methods Appl. Mech. Eng. 193, 1367 (2004)

${ }^{18}$ S. Ramakrishnan and S. S. Collis, "Multiscale modeling for turbulence simulation in complex geometries," AIAA Paper No. 2004-0241, Reno, Nevada, 5-8 January 2004.

${ }^{19}$ S. Ramakrishnan and S. S. Collis, "Variational multiscale modeling for turbulence control," AIAA 1st Flow Control Conference, AIAA Paper No. 2002-3280, St. Louis, Missouri, 24-26 June 2002.

${ }^{20}$ S. Ramakrishnan and S. S. Collis, "Turbulence control simulation using the variational multiscale method," AIAA J. 42, 745 (2004).

${ }^{21}$ S. Ramakrishnan and S. S. Collis, "Partition selection in multiscale turbulence modeling" (preprint).

${ }^{22}$ M. Germano, U. Piomelli, P. Moin, and W. H. Cabot, "A dynamic subgridscale eddy viscosity model," Phys. Fluids A 3, 1760 (1991).

${ }^{23}$ D. K. Lilly, "A proposed modification of the Germano subgrid-scale model," Phys. Fluids A 4, 633 (1992).

${ }^{24}$ M. Lesieur, "Large-eddy simulation: A tool for aeronautics," in Fluid Dynamics and Aeronautics-New Challenges, edited by J. Périaux, M. Champion, J. J. Gagnepain, O. Pironneau, B. Stoufflet, and P. Thomas (CIMNE, Barcelona, 2003), pp. 349-364. 